\title{
Archetypes and design
}

\author{
Viktor Pigulevskiy ${ }^{1,}$, and Liudmila Mirskaya ${ }^{1}$ \\ ${ }^{1}$ South Russian Humanitarian Institute, 344082, Rostov-on-Don, Russia,
}

\begin{abstract}
Identification of basic archetypes and their remote structure in the context of civilization design, industrial and communicative design makes it possible to specify age-long stability of certain patterns and instruments. This contributes to design of effective models and necessary things. Archetypes as constantly recurring patterns of actions reflected in the mind in the form of unconscious schemes, patterns of thinking, behaviour and perception. Archetypes are a level of connection between being and thinking in the process of work, an area of contact of human corporeality and material side of the world. In the context of technical progress, miniaturization of gadgets and integration of functions, archetypal items remain highly-demanded. The clock with a round dial symbolizes the archetype of "eternal return". Instruments and lever control systems, a lamp with the mechanism of "lever balance", which simulates levels of human arm freedom, express the archetype of action. A jar made by a master on a potter's wheel symbolizes a "vessel of life", embodies the archetype of rotation, "wheel of life". Symbolic figures of visual communication - circle, spiral, swastika and concentric circles - also present the archetype of rotation. Mandala, a special symbol that can be noticed in the design of visual communications, as well as in city plans in ancient civilizations, expresses the archetype of selfness. The design of civilizations has constants established by the contact of human corporeality and material side of the world. However, most of contemporary things that have a symbolic meaning lack being universal, since they are made within the structure of civilization, beyond incrustation of human corporeality in the world. Acting in the world, a person constantly repeats life patterns; this archetype of "eternal return" remains a basic attitude of the design.
\end{abstract}

\section{Introduction}

The theory of collective unconscious posed to philosophy an issue of archetypes, contents of the unconscious that not only project unconscious images into consciousness, but direct the practical life activity as well. Emergence of mass production and design-engineering makes it possible to formalize some aspects of human activity. The problem of design implies the use of archetypal models for creation of effective communication, comfortable life environment and efficient human labour. It is believed that identifying and linking

Corresponding author: 1ymirskaya@yandex.ru 
development with a suitable archetype increases the likelihood of its success (Lidwell, Holden, Butler, 2012). As a rule, when analysing people's lifestyle, ergonomic working conditions, creating tools and techniques, promoting a company or advertising, the archetypal images singled out by C.G. Jung are just laid on the subject being studied, which makes it possible to explain whether this way of brand development or advertisement strategy suits for this activity type. However, the method of "classifying according to categories" practically does not work in the objective world, especially when in the course of analysis there happens a confusion of the archetype, cultural image and symbol, and the archetype is improperly understood as a typical image, pattern, and representation (Mark, Pearson, 2005; Pendikova, 2012; Solovyev, 2014).

\section{Statement of problem and research method}

The purpose of this article is to identify archetypes in the field of design, which makes it possible to design efficient models and necessary things. It is important to differentiate between an archetype and other things - image, symbol and sign. The identification of design archetypes will make it possible to designate the age-long stability of certain patterns and instruments of civilization. In general, the problem can be formulated through the example of clock-dial - why under conditions of miniaturization growth and emergence of gadgets that combine a number of functions like a mobile phone (communication, internet, calculator, radio and watch) traditional clock with round dial does not fall out of use? The problem of existence of such things that seem to contradict technical progress and business requirements can be referred to archetypes in the mode of activity.

To solve the research problem, we use the activity theory according to which a person in the process of work changes, exploits the surrounding nature, creating "the second nature" (K. Marx) and at the same time develops his/her own creative abilities, of which goal setting is fundamental. Consequently, design becomes an expression of goal setting under the conditions of mass production.

\section{Archetypes and contact}

Archetypes of the collective unconscious are represented by human activity that was repeated million times, reflected in the form of unconscious schemes, patterns of thinking, behaviour and perception (Mirskaya, 2006). So far human activity is two-directional, so a person changing the world according to his/her goals not only changes the nature for practical reasons, but also changes his/her own abilities increasing "what knowledge" and "how knowledge". Thus, archetypes are a level of connection between being and thinking in the process of work, an area of contact of human corporeality and material side of the world. Correspondingly, the area of detection of archetypes is, on the one hand, depth psychology and, on the other hand, - things and tools used by a person "always" and "constantly". Mode of activity is the process of society activity in the context of recurring patterns of "always" and "constantly", as well as the process of person's activity in the cycle goal - means - result. Setting goals and using certain means of achievement, a person in the process of work adjusts his/her abilities and transforms the nature, creating a reasonable reality. This is the area of contact of human corporeality and material side of the world where an archetypal image is objectified and expressed in the sign and symbolic form.

\section{Clock and archetype of "eternal return"}


Going out of the mythological stage of cyclic time, a person starts measuring the current time of his/her activity. Specially organized environment and measurement in hours and calendar become conditions of a person's activity. Archaic time is cyclic, "eternal return" agricultural seasons and agrarian holidays that in the Middle Ages correlate with the Christian calendar (from Christmas, Easter, All Saints' Day etc.). The time of city life in the Middle Ages is regulated by church bells - time of work and leisure, prayers and holidays, fast and abstinence. If the calendar covers the year cycle, the clock presents division of the process within the finite period. Use of ancient observation of the sky together with universal human compulsion from depth of the mind to see correct forms presented as selfness in numbers 4 and 3 (C. Jung) makes it possible in combination of both to divide day time into 12 parts or a perfect Mandala. As night time falls out of active work, its measurement remains mythological, example of which can be Egyptian "The Book of the Dead". This discreteness is far from accuracy and is restricted by the method of measurement - ancient civilizations use sun, water, sand and fire clocks. Measurement of events is carried out with the help of numbers, since "time is the number of change in respect of before and after" (Aristotle). As it is known, the invention of mechanical clock takes place in the 14th century and the most accurate free-pendulum clock is made by Huygens (1658). In fact, only since the Renaissance accurate measurement of time comes into economic life, as commercial deals begin to develop and credit and financial relations start to form. From the 17th century, the human develops "sense of time" (Sunyagin, 1987). Thus, time as "onward motion" from the past to the future becomes the result of Progress concept generated by the Renaissance age. Firstly, accuracy of "business" division of the current time acquires a meaning, and then circadian cycle becomes a wheel of progress. Clock dial that gives a chance to know one's way around and act every day and in correlation with the calendar to plan events of the future life becomes a universal symbol of cycle of interrelation of the current present, "now", and daily recurring, "always". It is clear that a circle is a universal symbol of unity of different spheres of existence both spatial and temporal (eternity) (Toporov, 1994). Therefore, in the context of modern civilization, round dial preserves its functions, the main of which is planning of life for the nearest future. Digital clock that shows the immediate time is not effective in this respect and has a limited area of application.

\section{Instruments and action archetype}

Archetypes in a person's activity "work" in the area of contact of human body with material side of the world or culture artifacts. First of all, this is labour, which means using tools of trade in the goal-means-result scheme. The simplest tool since the time homo sapiens appeared is a stick as an extension of a hand. The body is not only fitted in the environment, but due to the tool it is incrusted in the flesh of the world: "Flesh, one can say, is a state of the body, but not the body in its anatomical and perceptive limitations; transgressive body, i.e. going over its limit by its affirmation" (Podoroga, 1995).

This simple tool means that operations with levers, spades, hammers, swords and other tools are archetypal. Besides habitus, experience of constantly developing skills and abilities, habits and automatic behaviour, we have a built-in way of action of the collective unconscious that is tried and tested through ages and can be specified as the archetype of action. But there are also buttons, switches, triggers adapted for controlling things with the help of fingers. Even when iPhone was created with gestures of sensor control it turned out that it was impossible to do without buttons, they had to be drawn on the display. Anglepoise lamp by George Carwardine (1932) becomes a typical expression of the archetype of action. However, it is not due to the fact that a complicated spring mechanism 
provides balance of the whole structure (Sudjic 2015). The following lamps Tizio by Richard Sapper (1972), Tolomeo by Michele de Lucchi (1987), Fortebraccio by Alberto Meda (1998), Archimoon by Philippe Starck (2000), Kelvin LED by Antonio Citterio (2010) do not have a spring mechanism, but they realize the same archetype. It is all about the design task set by George Carwardine, i.e. representation of degrees of freedom of a human arm action, which is just an "eternal" scheme that makes work of a human possible, makes it the archetype of action. Finally, archetypal projects are those where a person rotates something - steering wheels, handles, keys.

Peoples' habits to operate with things in a certain manner do not mean that the archetype works. The archetype of action should not be confused with abilities, skills, behaviour patterns or stereotypes of perception, i.e. mental patterns. The following are examples of patterns and stereotypes - psychological standard for a "qwerty" keyboard of a typewriter, habit to use hot and cold taps, gas burner knobs on the gas stove, and a traffic light as a conditional semantic system is a simple stereotype of perception (Norman, 2006). Nontypical installation of these things causes misunderstandings and mistakes with users.

Things that have functional purpose and acquire a symbolic meaning in culture do not necessarily have an archetypal unconscious way of action as the basis of their creation (Albus, 2004). For example, a chair as an object of contact of a human pose with the world. It acquires a practical meaning with cultural artifacts - interior, transport, architectural forms. A chair and an armchair are necessary things of technical civilization. Its functions extend from a working place to a comfortable leisure area, its symbolic meaning is to emphasize the status of a person: it is a throne where the emperor sits, a raised platform, where the teacher is among students, an armchair of the boss in the office. The chair does not appear in all cultures historically. In Chinese and Japanese cultures, a person sits in his/her bended knees or on his/her hunkers. This object built into the structure of modern civilization lacks being archetypally universal. Money also, whatever symbols it can bear, is the means of identification of the nation and the state, but it does not have a universal prototype. An archetype is expressed by symbols and mythologemes, however, the converse theorem is not true, few symbolic objects relate to archetypes in the structure of material world (Raizman, 2003).

\section{Jar and archetype of life}

There is a universal vessel from which it is customary to drink anything - a ceramic jar. Unlike identification features of bottles, a jar is multifunctional; it has a similar form in different cultures, i.e. a vessel for liquid, grain or corn with a narrow neck. With the invention of a potter's wheel in 3rd millennium BC, the jar gets an almost perfect shape. On the one hand, its shape allows it to be a vessel for storing food and cooking food on fire, embodying the civilization of "boiled" in contrast to natural "raw" (C. Levi-Strauss). Thus, a jar is connected with survival of a cultural human. On the other hand, the shape of a jar is embodiment of the master's hand who forms the substance (clay) and embodies the archetype of rotation. A jar, an amphora, a crater is a perfect archetypal object that also acts as a visual and tactile complex of the object of satisfying thirst, hunger and reproduction. Vessel of life is a symbolic meaning of a jar that hides the archetype of rotation.

\section{Mandala}

Mandala is a special symbol that can be seen in the design of visual communication as well as in the city plans in ancient civilizations; it expresses the archetype of Selfness. For 
example, visual perception of a moving object implies assessment of the vector and dynamics of the visual mass in relation to a hidden structure of a square by bisectors (Shapoval 2006). Regardless of a different layout of ancient cities - rectangular or radial, they all have a centre - "place for communication" - a round square with sacral centre. In case of mandala-plans, it is quite clear - ancient observation of the sky and sense of unity that is formed in human mind as a result of process of individuation, inner compulsion called Selfness. The geometric plan of mandala has not only an external space origin (vault of heaven), but also inner, mental origin.

Mandala can act as a "wheel of life". The archetype of rotation as a dynamic system is symbolically represented in the form of spiral, swastika, wheel, wheel cross, concentric circles. Clock with a round dial is a symbol of mandala. A spiral is cosmological starting with spiral galaxies to a whirlpool; its mythological meaning is formation and disappearance. Spirals or concentric circles can cause a hypnotic effect easily, which can be proved by posters of Saul Bass for A. Hitchcock film "Dizziness" (1958), "Offset Buch und Werbekunst, no.7" by Herbert Bayer, "Beethoven" (1955) by Josef Muller-Brockmann, "Nicolas" (1935) by Cassandre, "Races Gand Prix" (1953) by Max Huber (Heller, Ilic, 2008).

\section{Conclusion}

Thus, no scientific and technical progress offering integration of functions in devices and gadgets can cancel archetypal objects. Contrary to the established opinion, archetypes are not ever-present in design. As a rule, industrial production of things, where each massproduced thing is a print of technologies and factory cycle, has no relation to the archetypes. Using plenty of things is formation of habitus of a person, behavioural habits and stereotypes of perception, "knowledge of how" and "knowledge of what" that grow as experience of a civilized human. This also implies unconscious, automatic experience of activity that requires training and, as a result, it is not universal and eternal. At the same time, design of civilization has some constants determined by the contact of human corporeality and material side of the world. Acting in the world, a human constantly repeats patterns of behaviour in typical circumstances, so the archetype of "constant return" becomes a basic setting in design and architecture. Behind it we can see the images of the collective unconscious in production of life activity from craftwork to high-tech solutions.

\section{References}

1. W. Lidwell, K. Holden, J. Batler, Universal Principles of Design, SPb., 28 (2012).

2. M. Mark, C. Pearson, The Hero and the Outlaw. Building Extraordinary Brands through the Power of Archetypes (Moscow, 2005).

3. L. Mirskaya, Carl Gustav Jung (Rostov-on-Don, 2006).

4. D. Norman, The Design of Everyday Things, Moscow, 134-139, 226-234, 246, 254262 (2006).

5. I. Pendikova, L. Rakitina, Archetype and Symbol in Advertising (Moscow, 2012).

6. V. Podoroga, Phenomenology of the Body (Moscow, 1995).

7. D. Solovyev, Archetypes in Branding (Moscow,2014).

8. D. Sudjic, The Language of Things, Moscow, 57-64 (2015).

9. G. Sunyagin, Industrial Labour and the Renaissance Culture (L., 1987). 
10. V. Toporov, Geometric Symbols. Myths of the Peoples of the World. Encyclopaedia, M., 1, 272-273(1994).

11. A. Shapoval, Field Structure of the Image. Sociocultural Problems of Design, Antey, 12-17 (2006).

12. V. Albus, Icons of Design: The 20-th Century (Pestle Publishing Ltd, 2004).

13. S. Heller, M. Ilic, Icons of Graphic Design (L., 2008).

14. D. Raizman, History of Modern Design (L., 2003). 